\title{
Original
}

\section{Cáncer de mama contralateral y recurrencia en portadoras BRCA1/2 y no portadoras con alto riesgo de cáncer de mama hereditario tras mastectomía bilateral}

\author{
Marta Allué Cabañuz ${ }^{a, *}$, María Domingo Bretón ${ }^{b}$, Jorge Chóliz Ezquerro ${ }^{c}$, \\ María Dolores Arribas del Amo ${ }^{a}$ y Antonio Tomás Güemes Sánchez ${ }^{d}$ \\ ${ }^{a}$ Unidad de Mama, Hospital Clínico Universitario Lozano Blesa, Zaragoza, España \\ ${ }^{\mathrm{b}}$ Servicio de Cirugía General, Hospital Clínico Universitario Lozano Blesa, Zaragoza, España \\ ' Servicio de Cirugía General, Hospital Universitario Miguel Servet, Zaragoza, España \\ d Universidad de Zaragoza, Hospital Clínico Universitario Lozano Blesa, Zaragoza, España
}

INFORMACIÓN DEL ARTÍCULO

Historia del artículo:

Recibido el 18 de marzo de 2020

Aceptado el 7 de abril de 2020

On-line el $\mathrm{xxx}$

Palabras clave:

BRCA1

BRCA2

Cáncer de mama hereditario

Cáncer de mama contralateral

Recurrencia

\section{R E S U M E N}

Introducción: La mastectomía contralateral profiláctica (MCP) reduce el riesgo de cáncer contralateral en al menos un 90\%. Además, las portadoras de mutación BRCA tienen mayor riesgo de recurrencia ipsilateral y de un segundo tumor primario. El objetivo es evaluar el riesgo de cáncer contralateral y la recurrencia, y analizar factores predictivos en pacientes con cáncer de mama y mutaciones BRCA1/2 y no portadoras con alto riesgo de cáncer hereditario.

Métodos: Análisis observacional retrospectivo de 46 pacientes sometidas a mastectomía bilateral durante 2004-2018. Nueve pacientes BRCA1, 12 BRCA2 y 25 con alto riesgo sin mutación.

Resultados: Dieciséis pacientes con diagnóstico de novo y 30 tratadas previamente por cáncer de mama a las que realizamos MCP de manera diferida (en 10 de ellas por detección de mutación en BRCA a posteriori); mediana de seguimiento 79 meses. La técnica quirúrgica más usada fue la incisión lateral externa. En todas las pacientes se realizó reconstrucción inmediata.

En las piezas de MCP se encontraron 4 tumores in situ, 3 invasivos y una hiperplasia atípica. La incidencia de cáncer contralateral oculto fue del 15,2\%. Cinco pacientes presentaron recidiva 21,2 meses de media tras la intervención; SLE 83,74 meses y SG 84,33 meses. Los modelos de regresión identificaron mutación BRCA1/2 y alto riesgo sin mutación como factores predictivos significativos para tumor oculto, mientras que el tamaño tumo$\mathrm{ral} \geq 2 \mathrm{~cm}$ fue predictivo de recidiva.

Conclusiones: En nuestra serie 7 pacientes (15,2\%) habrían desarrollado un tumor contralateral en los años posteriores, y un 10,8\% presentaron recurrencia a pesar de MCP.

(C) 2020 AEC. Publicado por Elsevier España, S.L.U. Todos los derechos reservados.

\footnotetext{
* Autor para correspondencia.

Correo electrónico: martitaallue@hotmail.com (M. Allué Cabañuz).

https://doi.org/10.1016/j.ciresp.2020.04.008

0009-739X/C 2020 AEC. Publicado por Elsevier España, S.L.U. Todos los derechos reservados.
} 
Keywords:

BRCA1

BRCA2

Hereditary breast cancer Contralateral breast cancer Recurrence

\section{Contralateral breast cancer and tumor recurrence in BRCA1/2 carriers and non-carriers at a high risk of hereditary breast cancer after bilateral mastectomy}

\begin{abstract}
A B S T R A C T
Introduction: Contralateral prophylactic mastectomy (CPM) has been reported to reduce risk of contralateral breast cancer (CBC) by at least $90 \%$.In addition, BRCA carriers presents higher risk of ipsilateral recurrence and a second primary tumor.

The aim is to evaluate risk of $C B C$ and recurrence and to analyze predictive factors in BRCA1/2 mutation carriers and non-carriers at high-risk of hereditary breast cancer patients.

Methods: Retrospective observational study. 46 patients underwent bilateral mastectomy during 2004-2018.

Results: Cohort comprised 9 patients BRCA1,12 BRCA2 and 25 at high-risk without mutation. Median follow-up 79 months. 16 patients recently diagnosed and 30 previously treated by breast cancer whom underwent CPM at second time (because of later detection of BRCA mutation in 10 cases). The external lateral incision was most frequent surgical technique. In all patients immediate reconstruction was performed.

In CPM pieces, 4 in situ carcinoma, 3 invasive and 1 atypical hyperplasia were found. The incidence of occult contralateral cancer was $15.2 \%$. Recurrence was observed in 5 patients a media of 21.2 months after surgery. FSD was 83.74 months and OS 84.33 months. Regression models identified BRCA1/2 mutation and high risk without mutation as significant occult tumor predictive factors while tumor size $\geq 2 \mathrm{~cm}$ was predictive of recurrence.

Conclusions: In our series we found a10.8\% recurrence despite CPM and 7 patients (15.2\%) would have developed a CBC in subsequent years.
\end{abstract}

(C) 2020 AEC. Published by Elsevier España, S.L.U. All rights reserved.

\section{Introducción}

Hasta un 5-10\% de las pacientes con cáncer de mama son portadoras de al menos un gen de susceptibilidad, incluidos TP53, PTEN, LKB1, MSH2/MLH1 y BRCA1 y 2, siendo en la mayoría de casos por mutaciones en estos últimos ${ }^{1}$. Las mujeres con una mutación de la línea germinal en el gen BRCA1 o BRCA2 tienen un alto riesgo de desarrollar cáncer de mama a lo largo de su vida, del $57 \%$ y $49 \%$ respectivamente según un metaanálisis ${ }^{2}$. Además, tienen un riesgo 4,5 y 3,4 veces mayor de cáncer de mama contralateral $(\mathrm{CMC})^{3}$ y mayor riesgo de recurrencia ${ }^{4}$.

En estudios observacionales prospectivos la mastectomía profiláctica bilateral ha demostrado disminuir la incidencia de cáncer de mama en un $90 \%$ en pacientes con mutación BRCA, y mejoría en la calidad de vida de las pacientes al disminuir la ansiedad y el miedo a padecer cáncer de mama. En el caso de presentar un cáncer 2 factores se asociaron con disminución del 50\% de riesgo de CMC: salpingooforectomía bilateral en BRCA2 (RR: 0,52; IC 95\%: 0,37-0,74) y edad mayor a 40 años, y un factor adicional que reduce este riesgo es la utilización de tamoxifeno adyuvante (RR: 0,57: IC 95\%: 0,43-0,75) ${ }^{5}$. Sin embargo, los beneficios de la mastectomía profiláctica contralateral en estas pacientes han sido más discutidos. Una revisión sistemática Cochrane estableció que la mastectomía contralateral profiláctica (MCP) podía prevenir el aumento de riesgo de CMC sin impacto en la supervivencia ${ }^{6}$. Desde 2014 existe nueva evidencia que apoya un beneficio en la supervivencia global $(89 \% \text { vs. } 71 \%, \mathrm{p}<0,001)^{7,8}$, siendo independiente de la realización de salpingooforectomía, y especialmente en menores de 40 años, estadio 1 y 2, no triple negativos y mujeres no tratadas con quimioterapia ${ }^{9}$. Por este motivo parece razonable realizar mastectomía bilateral en pacientes con cáncer unilateral portadoras de mutaciones en genes BRCA $^{10}$.

El objetivo de este estudio es evaluar el riesgo de CMC y recurrencia y analizar factores predictivos en pacientes con cáncer de mama portadoras de mutaciones BRCA1/2 y no portadoras con alto riesgo de cáncer de mama hereditario.

\section{Métodos}

Análisis observacional retrospectivo: se incluyeron pacientes con cáncer de mama unilateral portadoras de mutaciones en los genes BRCA1 y 2 a las que se realizó mastectomía bilateral (es decir, una mastectomía contralateral profiláctica) durante el periodo 2004-2018.

Los criterios para realizar el test genético (BRCA1/2) fueron los siguientes:

\footnotetext{
- Edad al diagnóstico $\leq 40$ años.

- Pacientes con múltiples cánceres primarios.

- Pacientes con mutaciones BRCA conocidas en la familia.
} 
- Pacientes con 2 familiares de primer grado con cáncer de mama.

- Pacientes con un familiar de primer grado con cáncer bilateral y otro de segundo grado con historia de cáncer de mama.

- Pacientes con un familiar de primer grado con cáncer $<45$ años y otro de segundo grado con historia de cáncer de mama.

El consejo genético se implantó en nuestro centro en el año 2013. Hasta ese momento se ofrecía realizar la mastectomía contralateral profiláctica a las pacientes con un cáncer de mama y fuerte historia familiar de cáncer de mama en ausencia de mutación conocida.

Se excluyeron pacientes BRCA o no portadoras con alto riesgo que optaron por cirugía conservadora o por mastectomía unilateral, así como pacientes con cáncer unilateral no portadoras BRCA o sin alto riesgo por historia familiar sometidas a MCP por otros motivos (tipo de tumor, elección, etc.) y pacientes con cáncer bilateral sincrónico, ya que el propósito era evaluar el riesgo de CMC.

Se realizó mastectomía preservadora de piel y pezón con reconstrucción inmediata mediante colocación de implante de silicona definitivo en todos los casos. Se utilizaron 4 patrones: mastectomía mediante incisión lateral externa, patrón de Wise, técnica de Spira e incisión radial externa. La ausencia de enfermedad en la base del complejo aréola pezón fue comprobada mediante biopsia intraoperatoria.

\section{Análisis estadístico}

Análisis descriptivo de las variables a estudio: para variables cualitativas se aportan frecuencias relativas y absolutas, para variables cuantitativas media y desviación estándar. Para evaluar diferencias se emplearon los test estadísticos de Chi cuadrado o test de Fisher en el caso de variables cualitativas y test de U de Mann Whitney o «t» de Student para variables cuantitativas según criterios de normalidad. Se realizan modelos de regresión logística para identificar los factores predictivos de cáncer oculto y recidiva. Se evalúan la edad, el alto riesgo por antecedentes familiares, las mutaciones BRCA, el tamaño tumoral, la afectación ganglionar y la histología del cáncer de mama índice, multifocalidad y multicentricidad. Para evaluar la supervivencia global, supervivencia libre de enfermedad (SLE) y tasa de recidiva se utilizó el test de logrank. Se calculó la SLE como el tiempo desde la intervención quirúrgica hasta la aparición de recaída local o sistémica durante el período de estudio.

Se estableció el nivel de significación estadística para un valor de p menor a 0,05. Para todo el análisis se ha utilizado el programa estadístico SPSS 22.0 para Windows (SPSS Ibérica, Madrid, España).

Este estudio se adhiere a los principios éticos de la Declaración de Helsinki y fue aprobado por el comité ético del centro.

\section{Resultados}

Durante el periodo de estudio se incluyeron 46 pacientes. De ellas 9 pacientes $(19,6 \%)$ eran portadoras de mutación en BRCA1, 12 en BRCA2 $(26,1 \%)$ y 25 pacientes $(54,3 \%)$ con alto riesgo sin mutación. Un total de 16 pacientes (34,7\%) presentaban diagnóstico de novo y 30 (65,2\%) habían sido tratadas previamente por cáncer de mama, a las que realizamos la MCP de manera diferida (en 10 de ellas por detección de mutación en genes BRCA a posteriori). La edad media de las pacientes fue de 47,4 \pm 9,2 años. La distribución de las pacientes se expone en la figura 1.

Se realizó mastectomía bilateral con reconstrucción inmediata. La técnica más usada fue la incisión lateral externa $(43,5 \%, 20 / 46)$ seguida de la técnica de Spira $(28,3 \%, 13 / 46)$. Los datos demográficos y relativos a la técnica quirúrgica se exponen en la tabla 1. La tasa global de complicaciones fue del 21,7\% y ninguna paciente precisó reintervención.

La mediana de seguimiento fue de 79 meses (DE: 51,62).

De las 16 pacientes con un cáncer de nuevo diagnóstico la indicación de realizar la MCP simultánea fue determinada por tratarse de un tumor multicéntrico y/o multifocal en 3 casos, carcinoma ductal in situ en un caso, carcinoma lobulillar infiltrante en otro caso y elección de la paciente en los 11 restantes.

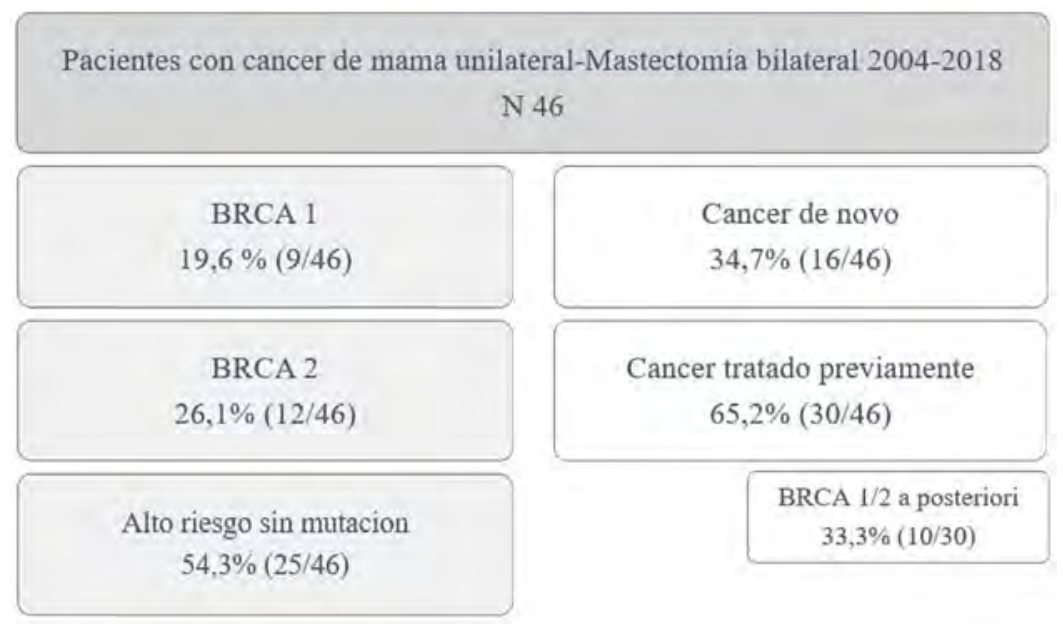

Figura 1 - Distribución de las pacientes incluidas en el estudio. 
Tabla 1 - Datos demográficos y relativos a la técnica quirúrgica

\begin{tabular}{|c|c|c|c|c|}
\hline & $\begin{array}{l}\text { Toda la serie } \\
\qquad \mathrm{N}=46\end{array}$ & $\begin{array}{c}\text { BRCA1 } \\
\mathrm{N}=9\end{array}$ & $\begin{array}{l}\text { BRCA2 } \\
\mathrm{N}=12\end{array}$ & $\begin{array}{l}\text { Alto riesgo sin mutación } \\
\qquad \mathrm{N}=25\end{array}$ \\
\hline Edad media & $47,4 \pm 9,2$ años & $43,5 \pm 8,1$ años & $44,2 \pm 6,6$ años & $48,7 \pm 6,2$ años \\
\hline Obesidad & $15,2 \%(7 / 46)$ & $11 \%(1 / 9)$ & $16,6 \%(2 / 12)$ & $16 \%(4 / 25)$ \\
\hline Tabaquismo & $6,5 \%(3 / 46)$ & 0 & $8,3 \%(1 / 12)$ & $8 \%(2 / 25)$ \\
\hline \multicolumn{5}{|l|}{ Técnica quirúrgica } \\
\hline Incisión lateral externa & $43,5 \%(20 / 46)$ & $33,3 \%(3 / 9)$ & $58,3 \%(7 / 15)$ & $40 \%(10 / 25)$ \\
\hline Wise corto & $23,9 \%(11 / 46)$ & $33,3 \%(3 / 9)$ & $33,3 \%(4 / 12)$ & $16 \%(4 / 25)$ \\
\hline Spira & $28,3 \%(13 / 46)$ & $33,3 \%(3 / 9)$ & $8,3 \%(1 / 12)$ & $36 \%(9 / 25)$ \\
\hline Incisión radial externa & $4,3 \%(2 / 46)$ & 0 & 0 & $8 \%(2 / 25)$ \\
\hline Complicaciones & $21,7 \%(10 / 46)$ & $11 \%(1 / 9)$ & $8,3 \%(1 / 12)$ & $32 \%(8 / 25)$ \\
\hline
\end{tabular}

En el grupo de pacientes tratadas previamente por un tumor se realizó la MCP de manera diferida; en 10 casos al detectarse $a$ posteriori la mutación en genes BRCA, en 2 casos por recidiva, en 6 por márgenes afectos tras tumorectomía (y preferencia de la paciente de realizarse MB en lugar de ampliación de márgenes), en 6 casos por tratarse de CLI, 3 carcinomas ductales in situ y 3 tumores multicéntricos/multifocales.

El tamaño tumoral medio fue de $1,65 \mathrm{~cm}(0,1-7)$. En 12 pacientes existía afectación ganglionar clínica al diagnóstico, por lo que se practicó linfadenectomía axilar (LA) y biopsia selectiva de ganglio centinela del lado contralateral (MCP). De ellas solo 4 fueron positivas (4/12, 33\%). Del resto de pacientes a las que se practicó biopsia selectiva del ganglio centinela únicamente fue positivo en 4 casos, realizando entonces LA, con resultado positivo únicamente en 2 casos. Los datos clínicos y patológicos relativos al tumor se exponen en la tabla 2.

En las piezas de MCP se encontraron 4 tumores in situ (CLIS), 3 invasivos (CDI) y una hiperplasia atípica. La incidencia de cáncer contralateral oculto fue del 15,2\% (7/46) $(p=0,021)$.

Cinco pacientes presentaron recidiva (2 local y 3 a distancia), con una media de 21,2 meses (rango 1-59) tras la intervención, y 3 fallecieron. La tasa de recidiva fue del 10,8\% (5/46). El tiempo medio de SLE fue de 83,74 meses y supervivencia global 84,33 meses. Los datos se detallan en la tabla 3.

Tabla 2 - Datos clinicopatológicos relativos al tumor

\begin{tabular}{|c|c|c|c|}
\hline & $\begin{array}{c}\text { Toda la serie } \\
\qquad N=46\end{array}$ & $\begin{array}{l}\text { Cáncer de novo } \\
\qquad N=16\end{array}$ & $\begin{array}{c}\text { Cáncer previo } \\
\qquad \mathrm{N}=30\end{array}$ \\
\hline \multicolumn{4}{|l|}{ Indicación $\mathrm{MCP}$} \\
\hline CDIS & $4(8,7 \%)$ & $1(6,3 \%)$ & $3(10 \%)$ \\
\hline CLI & $7(15,2 \%)$ & $1(6,3 \%)$ & $6(20 \%)$ \\
\hline MF/MC & $6(13 \%)$ & $3(18,8 \%)$ & $3(10 \%)$ \\
\hline Elección sin otro factor & $11(23,9 \%)$ & $11(23,9 \%)$ & 0 \\
\hline Margen afecto tras tumorectomía & $6(13 \%)$ & 0 & $6(20 \%)$ \\
\hline Recidiva tras tumorectomía & $2(4,3 \%)$ & 0 & $2(6,7 \%)$ \\
\hline BRCA detectado a posteriori & $10(21,7 \%)$ & 0 & $10(33,3 \%)$ \\
\hline \multicolumn{4}{|l|}{ Datos relativos al tumor índice } \\
\hline Tamaño tumoral & $1,55 \mathrm{~cm}(0,1-7)$ & $1,75 \mathrm{~cm}(0,2-7)$ & $1,4 \mathrm{~cm}(0,1-4)$ \\
\hline $\mathrm{N}$ positiva al diagnóstico & $26 \%(12 / 46)$ & $50 \%(8 / 16)$ & $13,3 \%(4 / 30)$ \\
\hline LA positiva & $33,3 \%(4 / 12)$ & $37,5 \%(3 / 8)$ & $25 \%(1 / 4)$ \\
\hline
\end{tabular}

Tabla 3 - Resultados oncológicos

\begin{tabular}{lllll} 
& $\begin{array}{c}\text { Toda la serie } \\
\mathrm{N}=46\end{array}$ & $\begin{array}{c}\text { BRCA1 } \\
\mathrm{N}=9\end{array}$ & $\begin{array}{c}\text { BRCA2 } \\
\mathrm{N}=12\end{array}$ & \multicolumn{1}{c}{$\begin{array}{c}\text { Alto riesgo sin mutación } \\
\mathrm{N}=25\end{array}$} \\
\hline Hallazgos anatomopatológicos en piezas de mastectomía contralateral profiláctica & & \\
Tumor oculto & $15,2 \%(7 / 46)$ & $11 \%(1 / 9)$ & $25 \%(3 / 12)$ & $12 \%(3 / 25 \%)$ \\
$\quad$ CDIS & $8,7 \%(4 / 46)$ & $11 \%(1 / 9)$ & $25 \%(3 / 12)$ & 0 \\
Cáncer invasivo & $6,5 \%(3 / 46)$ & 0 & 0 & $12 \%(3 / 25)$ \\
HP atípica & $2,2 \%(1 / 46)$ & 0 & 0 & $4 \%(1 / 25)$ \\
Benignidad & $82 \%(38 / 46)$ & $77,8 \%(7 / 9)$ & $50 \%(6 / 12)$ & $72 \%(18 / 25)$ \\
Resultados oncológicos & & & & \\
Recidiva & $10,8 \%(5 / 46)$ & $11 \%(1 / 9)$ & $8,3 \%(1 / 12)$ & $12 \%(3 / 25)$ \\
Recidiva local & $4,3 \%(2 / 46)$ & $11 \%(1 / 9)$ & 0 & $4 \%(1 / 25)$ \\
Recidiva a distancia & $6,5 \%(3 / 46)$ & 0 & $8,3 \%(1 / 12)$ & $8 \%(2 / 25)$ \\
SLE media meses (DS) & $83,74(55,81)$ & $89,5(60,6)$ & $84,9(58,1)$ & $76,6(52,3)$ \\
SG media meses (DS) & $84,33(55,2)$ & $90,4(59,6)$ & $85,7(56,7)$ & $73,6(48,1)$ \\
\hline
\end{tabular}


Tabla 4 - Modelos de regresión univariante. Se muestran únicamente resultados con significación estadística

\begin{tabular}{lcc} 
& $\begin{array}{c}\text { Tumor oculto } \\
\text { OR (IC 95\%) }\end{array}$ & $\begin{array}{c}\text { Recidiva } \\
\text { OR (IC 95\%) }\end{array}$ \\
\hline $\begin{array}{l}\text { Edad }<40 \text { años } \\
\text { Edad }>40 \text { años }\end{array}$ & \\
Alto riesgo sin mutación & $2,529(1,230-5,199)$ & \\
Mutaciones BRCA1/2 & $2,892(1,275-6,561)$ & \\
Tamaño tumoral $>2 \mathrm{~cm}$ & & $5,01(2,06-16,22)$ \\
Afectación ganglionar & & \\
CDIS & & \\
CLIS & & \\
Cáncer invasivo & \\
MF/MC & \\
\hline
\end{tabular}

Finalmente se realizó análisis univariante y los modelos de regresión identificaron las mutaciones BRCA1/2 y alto riesgo sin mutación como factores predictivos significativos para tumor oculto (OR = 2,892 [IC 95\%: 1,275-6,561] $p=0,003)(2,529$ $[1,230-5,199] \mathrm{p}=0,004)$ mientras que el tamaño del tumor $\geq 2 \mathrm{~cm}$ fue predictivo de recidiva (OR = 5,01 [IC 95\%: 2,06-16,22] $\mathrm{p}=0,002)$ (tabla 4).

\section{Discusión}

Es un hecho reconocido que las pacientes con antecedentes de cáncer de mama tienen un riesgo de 1,5 a 2 veces mayor de desarrollar CMC que la población general ${ }^{11}$, y todavía es mayor en las portadoras de mutaciones en genes BRCA1 y BRCA2 de 4,5 y 3,4 veces mayor respectivamente ${ }^{3}$.

Metcalfe et al. estiman en su estudio un riesgo a 10 años de CMC del $23,8 \%$ para BRCA1 y del $18,7 \%$ para BRCA $2{ }^{12}$, lo cual es consistente con nuestros resultados. En nuestro estudio se detecta una tasa de tumor oculto del $15,2 \%$, siendo más de la mitad tumores in situ que tardarían tiempo en demostrarse clínicamente.

Existen varios factores que han demostrado influir en el riesgo de CMC en portadores de mutaciones BRCA con cáncer de mama como la edad temprana al diagnóstico ${ }^{12}$, habiéndose evidenciado un aumento del riesgo relativo a medida que aumenta la edad al diagnóstico ${ }^{3}$. En nuestra serie la edad menor a 40 años no se asoció significativamente con un mayor riesgo de presentar CMC.

El objetivo del tratamiento en pacientes con cáncer de mama es minimizar el riesgo de muerte por ese tumor, sin embargo, en las pacientes con mutaciones en genes BRCA o alto riesgo de cáncer de mama hereditario sin mutación conocida debemos además minimizar la incidencia y mortalidad debidas a cánceres posteriores que se puedan desarrollar. Así, la detección intensiva puede ayudar a diagnosticar tumores metacrónicos en estadios precoces, pero no puede prevenir el desarrollo de los mismos. Por tanto, se deben plantear estrategias preventivas como la MCP, que en pacientes de riesgo reduce el riesgo de CMC en al menos un $90 \%{ }^{13}$. En nuestra serie, 7 pacientes $(15,2 \%)$ habrían desarrollado un tumor contralateral en los años posteriores.

Por otra parte, entre las pacientes con cáncer de mama tratadas con cirugía conservadora, las portadoras de mutación
BRCA tienen un mayor riesgo de recurrencia ipsilateral y de desarrollar un segundo tumor primario, en comparación con los controles esporádicos ${ }^{11}$. En un estudio multicéntrico Pierce et al. ${ }^{14}$ describen una tasa de recurrencia ipsilateral a 10 años 2 veces mayor en pacientes BRCA, y en el estudio de Metcalfe $\mathrm{e}^{12}$ se cuantifica en un riesgo del $11 \%$ para mujeres con una mutación BRCA1 y del 17\% para BRCA2, lo que corresponde con nuestros resultados (tasa de recidiva del 10,8\%). Sin embargo, no se han encontrado diferencias significativas en el desarrollo de recurrencia entre las portadoras de mutación BRCA1/2 y las no portadoras de alto riesgo, lo que probablemente se deba a que se han comparado las portadoras de mutación BRCA con las no portadoras de alto riesgo y no con pacientes con cáncer de mama esporádico.

Este estudio presenta las limitaciones propias del diseño retrospectivo de centro único. El pequeño número de pacientes en cada subgrupo se traduce en un poder estadístico débil y un IC amplio. Durante el periodo recogido existieron cambios sustanciales como la creación de una consulta de consejo genético que permitió detectar más pacientes con mutaciones de riesgo, además de implantar otras medidas de prevención como la salpingooforectomía profiláctica, lo que se traduce en nuestros resultados, y posiblemente en años próximos la tasa de detección de cáncer oculto disminuya.

En conclusión, los resultados de este estudio muestran que tanto las portadoras de mutación BRCA1/2 como las no portadoras con alto riesgo de cáncer de mama hereditario tienen un alto riesgo de desarrollar CMC y recurrencia.

Se debe considerar el asesoramiento para llevar a cabo medidas preventivas como la MCP independientemente del estado de mutación BRCA1/2 en pacientes de alto riesgo, fundamentalmente determinado por la historia familiar.

\section{Conflicto de intereses}

No existe conflicto de intereses.

\section{Agradecimientos}

A los integrantes de Unidad de Mama del Hospital Clínico Universitario Lozano Blesa de Zaragoza, cirujanos, personal de enfermería y a las pacientes.

\section{B I B L I O G R A F Í A}

1. Robson ME, Boyd J, Borgen PI, Cody HS 3rd. Hereditary breast cancer. Curr Probl Surg. 2001;38:387-480.

2. Chen S, Parmigiani G. Meta-analysis of BRCA1 and BRCA2 penetrance. J Clin Oncol. 2007;25:1329-33.

3. Malone KE, Begg CB, Haile RW, Borg A, Concannon P, Tellhed $\mathrm{L}$, et al. Population-based study of the risk of second primary contralateral breast cancer associated with carrying a mutation in BRCA1 or BRCA2. J Clin Oncol. 2010;28:2404-10.

4. Garcia-Etienne CA, Barile M, Gentilini OD, Botteri E, Rotmensz N, Sagona A, et al. Breast-conserving surgery in BRCA1/2 mutation carriers: Are we approaching an answer? Ann Surg Oncol. 2009;16:3380-7. 
5. Metcalfe K, Lynch HT, Ghadirian P, Tung N, Olivotto I, Warner E, et al. Contralateral breast cancer in BRCA1 and BRCA2 mutation carriers. J Clin Oncol. 2004;22:2328-35.

6. Lostumbo L, Carbine NE, Wallace J. Prophylactic mastectomy for the prevention of breast cancer. Cochrane Databasde sys Rev. 2010;10. CD002748. doi: 10.1002/14651858.

7. Evans DG, Ingham SL, Baildam A, Ross GL, Lalloo F, Buchan I, et al. Contra-lateral mastectomy improves survival in women with BRCA1/2-associated breast cancer. Breast Cancer Res Treat. 2013;140:135-42.

8. Metcalfe K, Gershman S, Ghadirian P, Lynch HT, Snyder C, Tung N, et al. Contralateral mastectomy and survival after breast cancer in carriers of BRCA1 and BRCA2 mutations: Retrospective analysis. BMJ. 2014;348:226.

9. HeemskerkGerritsen BA, Rookus MA, Aalfs CM, Ausems MG, Collee JM, Jansen L, et al. Improved overall survival after contralateral risk-reducing mastectomy in BRCA1/2 mutation carriers with a history of unilateral breast cancer: A prospective analysis. Int J Cancer. 2015;136:668-77.
10. Domchek SM, Friebel TM, Singer CF, Evans DG, Lynch HT, Isaacs $\mathrm{C}$, et al. Association of risk-reducing surgery in BRCA1 or BRCA2 mutation carriers with cancer risk and mortality. JAMA. 2014;304:967-75.

11. Kang E, Kim SW. The Korean hereditary breast cancer study: Review and future perspectives. J Breast Cancer. 2013;16:245-53.

12. Metcalfe K, Gershman S, Lynch HT, Ghadirian P, Tung N, Kim-Sing C, et al. Predictors of contralateral breast cancer in BRCA1 and BRCA2 mutation carriers. Br J Cancer. 2011;104:1384-92.

13. Valachis A, Nearchou AD, Lind P. Surgical management of breast cancer in BRCA-mutation carriers: A systematic review and meta-analysis. Breast Cancer Res Treat. 2014;144:443-55.

14. Pierce LJ, Levin AM, Rebbeck TR, Ben-David MA, Friedman E, Solin LJ, et al. Ten-year multi-institutional results of breastconserving surgery and radiotherapy in BRCA1/2-associated stage I/II breast cancer. J Clin Oncol. 2006;24:2437-43. 